\title{
Product Quality Monitoring in Hydraulic Presses Using a Minimal Sample of Sensor and Actuator Data
}

\author{
IRIS WEISS, BIRGIT VOGEL-HEUSER, EMANUEL TRUNZER, and SIMON KRUPPA, \\ Technical University of Munich
}

\begin{abstract}
Machine learning and artificial intelligence provide methods and algorithms to take advantage of sensor and actuator data in automated production systems. Product quality monitoring is one of the promising applications available for data-driven modeling, particularly in cases where the quality parameters cannot be measured with reasonable effort. This is the case for defects such as cracks in workpieces of hydraulic metal powder presses. However, the variety of shapes produced at a powder press requires training of individual models based on a minimal sample size of unlabeled data to adapt to changing settings. Therefore, this article proposes an unsupervised product quality monitoring approach based on dynamic time warping and non-linear regression to detect anomalies in unlabeled sensor and actuator data. A preprocessing step that isolates only the relevant intervals of the process is further introduced, facilitating efficient product quality monitoring. The evaluation on an industrial dataset with 37 samples, generated in test runs, shows a truepositive rate for detected product quality defects of $100 \%$ while preserving an acceptable accuracy. Moreover, the approach achieves the output within less than 10 seconds, assuring that the result is available before the next workpiece is processed. In this way, efficient product quality management is possible, reducing timeand cost-intensive quality inspections.
\end{abstract}

CCS Concepts: • Computing methodologies $\rightarrow$ Machine learning; Learning paradigms; Unsupervised learning; Anomaly detection;

Additional Key Words and Phrases: Product quality monitoring, hydraulic metal powder press, unsupervised machine learning, minimal sample size, cyber-physical system

\section{ACM Reference format:}

Iris Weiss, Birgit Vogel-Heuser, Emanuel Trunzer, and Simon Kruppa. 2021. Product Quality Monitoring in Hydraulic Presses Using a Minimal Sample of Sensor and Actuator Data. ACM Trans. Internet Technol. 21, 2, Article 37 (April 2021), 23 pages.

https://doi.org/10.1145/3436238

The project "Online Knowledge Management, Data-Mining and Machine Learning in Mechanical Engineering" (M@OK) received funding by the Bavarian Ministry of Economic Affairs, Energy and Technology (StMWi) under grant number IUK566.

Authors' address: I. Weiss, B. Vogel-Heuser, E. Trunzer, and S. Kruppa, Institute of Automation and Information Systems, Technical University of Munich, Munich, Germany; emails: \{iris.weiss, vogel-heuser, emanuel.trunzer, simon.kruppa\}@tum.de.

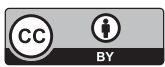

This work is licensed under a Creative Commons Attribution International 4.0 License

(C) 2021 Copyright held by the owner/author(s).

1533-5399/2021/04-ART37

https://doi.org/10.1145/3436238

ACM Transactions on Internet Technology, Vol. 21, No. 2, Article 37. Publication date: April 2021. 


\section{INTRODUCTION TO MACHINE LEARNING FOR PRODUCT QUALITY MONITORING}

Technologies of Industrie 4.0 (I4.0) reveal the significant potential in optimizing automated systems based on self-aware and intelligent functionalities (the so-called fourth industrial revolution). The result is a cyber-physical system (CPS), which is more flexible and adaptable under changing environmental conditions. Besides the communication technology, which transfers and processes the gathered data and information in CPS, machine learning and artificial intelligence algorithms are prerequisites for I4.0 applications [1]. The impact of such data-driven I4.0 applications is often quantified with the increase of the overall equipment effectiveness, including the factors of availability, performance, and quality. Therefore, product quality monitoring in CPS is the focus of numerous research activities. However, an increase in quality should not affect the performance negatively. Complex measurements of product quality, increasing cycle times during production, cannot be an efficient solution. This article focuses on data-driven monitoring to replace time- and cost-intensive quality inspection systems to improve quality monitoring. An approach based on sensor and actuator data of a hydraulic metal powder press (HMPP) is proposed to monitor quality criteria such as cracks in the workpieces that are not observable during production with reasonable effort. The challenges of such a quality monitoring approach are discussed in detail in the following section.

\subsection{Challenges of Product Quality Monitoring in HMPPs}

Hydraulic presses are used to influence the characteristics of a material such as shape and density based on high forces. One particular use case is the compaction of metal powder. In powder metallurgy, which describes a variety of approaches to produce solid workpieces, three different technological processes are involved: powder production, powder compaction, and sintering. To understand the requirements for a product quality monitoring approach for HMPP, a short introduction to powder metallurgy is given based on ASM International [2]. Powder compaction is a mechanical process to press powder and use the mechanical interlock of particles to receive a porous and shaped workpiece. Sintering is the subsequent heat treatment that changes the chemical and mechanical characteristics to receive the required solid workpiece. Commonly used technologies for powder compaction of metal powder are hydraulic presses. State-of-the-art HMPPs are highly automated machines following the principle demonstrated in Figure 1(a). With axial movements of different punches, geometric shapes such as gear wheels can be produced (cf. Figure 1(b)). Forces up to $700 \mathrm{MPa}$ are possible. This powder compaction process can be divided into multiple consecutive phases. First, the powder is filled in the mold (filling phase). Subsequently, the punches perform the axial movement and the pressure is applied (pressing phase). Last, depressurizing denotes the removal of the punches (depressurizing phase) and demolding denotes the ejection of the workpiece (demolding phase).

With high precision, a workpiece is processed approximately every 10 seconds by the considered HMPP. Even though these state-of-the-art HMPPs are highly reliable, deviations in the process can lead to rare quality defects of the produced workpiece. Typical defects in powder compaction are cracks in the workpieces that usually occur during depressurizing and demolding. Cracks are caused by residual stress and tensions between workpiece and mold reducing the strength of the workpiece significantly. This is considered a severe quality defect. However, this latent quality issue can be reliably evaluated only after sintering with a visual inspection under black light. Even though the powder compaction process is identified as the reason for cracks, the quality inspection is not possible during or immediately after it. Furthermore, classical approaches of statistical process control reach their limits since the monitoring of single parameters based on statistical 

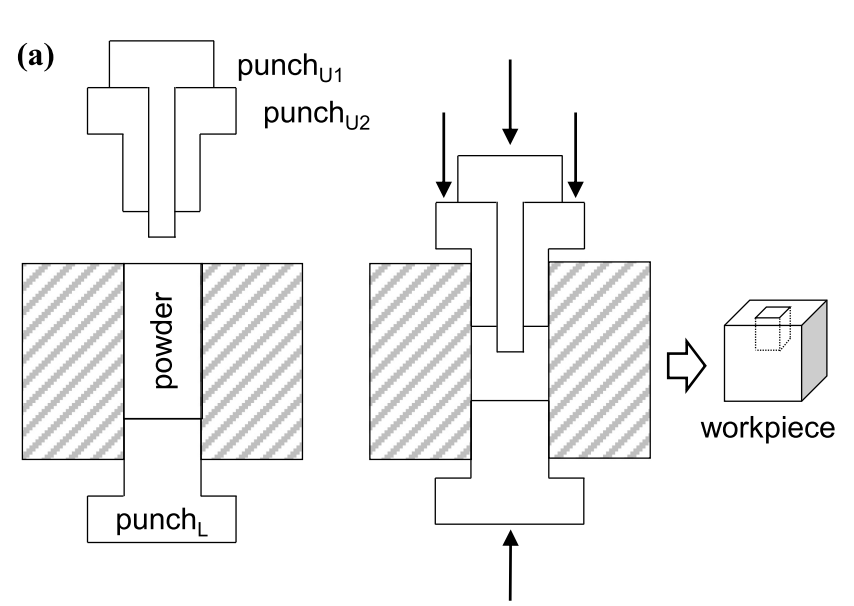

(b)

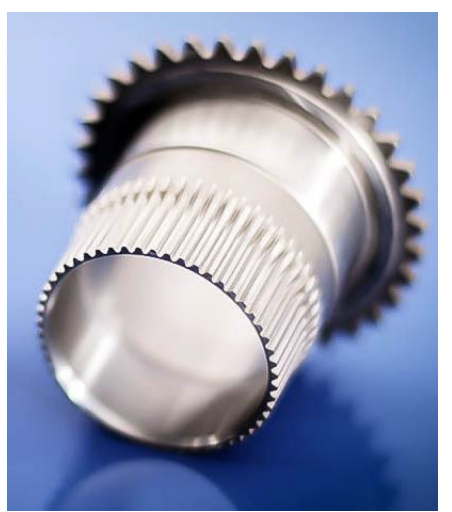

Fig. 1. (a) Schematic figure of the powder compaction process in HMPP. (b) Gear wheel produced at an HMPP.

measures as mean and standard deviations cannot cover the complex dependencies in such CPSs [3]. Therefore, a data-driven approach for product quality monitoring during or immediately after powder compaction is highly appreciated to reduce the effort of time- and cost-intensive quality inspections.

Sensor and actuator data of the considered HMPP is available representing the position signals of all punches and the resulting forces. Deviations of these signals from the expected signal path could serve as an indication for abnormal behavior causing quality defects. This is the case not only for HMPPs but also for a variety of hydraulic presses calling for a flexible and scalable product quality monitoring approach. Since no data is available upfront-that is, connecting cracks in workpieces with the sensor and actuator data during powder compaction-the development of a data-driven approach arises as an unsupervised learning task (requirement R1). Based on the unlabeled data, only anomaly detection can be performed rather than a prediction of product quality. Nonetheless, a quality monitoring approach should be able to isolate the detected anomaly in different types (requirement R2). Characteristics such as the time when the anomaly occurred or how long the anomaly lasted serve as criteria to distinguish among different types of anomalies. Based on this information, the workpiece is sorted out of the production process and a manual quality inspection is performed to confirm the defect and initiate rework. Since the workpiece's shape determines the arrangement of the punches (cf. Figure 1(a)), the dependencies of the sensor and actuator data change with every new shape. For this reason, a one-size-fits-all approach will not be effective and a new data-driven model is required for each product with a changed shape (requirement R3). To keep the training phase of a quality-monitoring model at the beginning of each batch as short as possible, the approach is required to perform effectively despite a minimal sample of the sensor and actuator data. Furthermore, an approach with low computational complexity is required to match the fast production process of a few seconds.

This article proposes an approach for product quality monitoring in HMPP to detect cracks in workpieces. Based on the sensor and actuator data (position and force), differentiation between normal behavior resulting in high-quality products and abnormal behavior causing quality defects is possible with a high true-positive rate (hypothesis 1). In this setting, the true-positive rate is given more importance than a low false-positive rate since warranty costs for delivered defective workpieces are huge (particularly for sectors such as automotive suppliers). Furthermore, the 
quality monitoring approach processes the data in a limited time span, enabling the assessment of the workpieces' conditions immediately after pressing (hypothesis 2).

The main contribution of this work is the development of a product quality monitoring approach for HMPP based on anomaly detection algorithms observing the signal paths of sensor and actuator data. The approach tackles the challenge of unlabeled data of minimal sample sizes in changing conditions (shape of workpieces). Algorithms, introduced in other disciplines, are adapted to the requirements of HMPPs to introduce a flexible and scalable monitoring approach with fast computation. This approach will increase the overall equipment effectiveness of the HMPP due to the changed strategy in quality monitoring. Without the data-driven approach, random samples, such as one in a hundred workpieces, are examined in time- and cost-intensive quality inspections. High-quality workpieces may be examined unnecessarily, while it still bears the danger that uninspected workpieces show quality defects, which stay hidden. In contrast, the proposed data-driven quality monitoring offers the advantage of targeted quality inspections. Only workpieces that show anomalies in the production process are examined. Consequently, time- and cost-intensive quality inspections are reduced while ensuring precise quality monitoring.

\subsection{Structure of the Article}

The rest of the article is structured as follows. In Section 2, the state of the art in data-driven product quality monitoring is summarized. The research of the past years is discussed based on two categories: supervised and unsupervised methods. In Section 3, the concept of the monitoring approach is elaborated, fulfilling the requirements of the given use case. Besides two different algorithms that will be compared, the preprocessing of the data is discussed. In the following, the evaluation of an industrial dataset is performed. Section 4.1 gives a descriptive overview of the available dataset, whereas Section 4.2 presents the results of the data preprocessing. The evaluation of the algorithms for product quality monitoring is conducted in Section 4.3. Finally, the article concludes in Section 5 with the summary and discussion of the effectiveness of the proposed monitoring approach. Furthermore, an outlook to future work in deploying this approach is given.

\section{STATE OF THE ART IN DATA-DRIVEN PRODUCT QUALITY MONITORING}

Product quality monitoring (also product quality prediction) is one of the promising applications available for data-driven approaches, including big data analysis, machine learning, and artificial intelligence [4]. The different approaches introduced in research can be classified based on the characteristics of the available data. Classification and regression models can be used for supervised learning tasks, meaning that process data and the corresponding product quality data is available for model training. Classification is used for categorical data (e.g., good vs. bad quality), whereas regression is used for continuous data. In contrast, clustering or anomaly detection models can be applied for unsupervised learning tasks. In clustering, the process data is grouped based on the characteristics of the data itself. The corresponding product quality data is not required for training. In anomaly detection, the process data is used to model the observed system behavior to detect deviations of it, which might correlate with quality defects. In the following, an overview is given describing the state of the art in supervised and unsupervised quality monitoring. Furthermore, the state of the art in HMPP is emphasized.

In industrial applications, support vector machines are often used for classification, whereas artificial neural networks are used for regression. Furthermore, fuzzy logic and evolutionary computation are applied frequently [4]. Since supervised learning methods are widely used for quality monitoring, the following paragraph gives just examples of the state of the art. In Kirchen et al. [5], a linear regression model is introduced for a multi-stage forming process. The regression 
model predicts the quality of the final product right from the beginning of the process, refining the prediction during each stage of the forming process. A non-linear regression approach, namely non-linear Gaussian mixture regression, is used for quality prediction in multimode processes [6]. These mixture models take into account that the dependencies and relations differ around the different modes of a process. Li et al. [7] propose a combination of the linear partial least-squares method with a support vector machine to cover non-linear dependencies for quality prediction in the Tennessee-Eastman process. Besides the mentioned regression approaches, neural networks are widely used for quality monitoring in industrial use cases. For the quality prediction of steel coils, such as yield and tensile strength, a neural network based on 23 process parameters is trained in Lalam et al. [8]. Gonzalez-Val et al. [9] proposed a convolutional neural network to detect quality defects in laser manufacturing. In contrast to the research approach of Lalam et al. [8], GonzalezVal et al. [9] used high-speed thermal images as input for the network rather than process data. Another approach for quality monitoring in laser welding is proposed by Shevchik et al. [10]. They introduce a support vector machine to detect the two main defects of laser welding based on the input of optical sensors. An extension for support vector machines to take the costs of quality monitoring into account is introduced in Oh et al. [11]. Warranty costs and rework costs are considered to develop cost-effective quality monitoring approaches. Extensive research has been performed in the past years to develop quality monitoring systems for manufacturing processes based on supervised learning methods. However, the considered process in this article requires unsupervised learning methods since no quality data is available for model training.

For unsupervised product quality monitoring, other methods have to be considered, which model normal behavior and detect abnormal situations. Clustering, particularly recurrent hierarchical Dirichlet process clustering, is used to identify process anomalies in semiconductor chemical mechanical planarization/polishing [12]. Sensor data such as vibration is used to detect and identify these anomalies. However, the computational complexity of this approach is high and unsuitable for the considered quality monitoring approach in this article. A widely used clustering algorithm for anomaly detection is the one-class support vector machine. Tan et al. [13] introduce a combination of several one-class support vector machines to detect faults as well as identify the degree of the faults. Xiao et al. [14] propose a robust version of this algorithm to avoid misclassification based on outliers in the training dataset. The algorithm is evaluated on the TennesseeEastman Process, detecting anomalies and faults, respectively. However, the well-established one-class support vector machine does not consider the temporal behavior of the signals. Therefore, the algorithm is not appropriate for the tasks of quality monitoring in hydraulic presses.

Besides the mentioned research, work that gives particular emphasis on hydraulic presses is discussed in the following. In Yu et al. [15], a model-based approach is introduced to monitor the stress and strain of a hydraulic press. Based on the comparison between sensor values and a finite element simulation, abnormal states are detected. This model-based approach is effective but also cost intensive. This also holds for the approach in Chen et al. [16]. A model-based approach is proposed, comparing mathematical models of stress and moving beam attitude with measured signals to monitor the state of the hydraulic press. Besides the drawbacks of model-based approaches, the two preceding works only observe the condition of the machine without connecting the results to product quality issues. Further research focuses on specific devices of a hydraulic press, such as the work of Sowmiya [17], which proposes the monitoring of the induction motor of a hydraulic press. In addition, quality monitoring in hydraulic presses is also subject to research. Ferreira et al. [18] introduce a design, including hardware and software, for a monitoring approach. In particular, a vision system is proposed measuring the springback of aluminum sheets based on images of the sheet. Ripanu et al. [19] perform a qualitative and quantitative (via simulations) analysis to develop a cause and effect representation of the parameters of a hydraulic press. They reveal the 
demand for monitoring chips and cracks during the stamping process of cage windows for bearing cages. Du et al. [20] propose a user interface to display critical key parameters to enable the operator to monitor the system efficiently. However, the mentioned work misses the potential of using machine learning based on sensor and actuator data to detect anomalies and quality defects automatically. Wang et al. [3] introduce a quality monitoring system for metal powder compaction based on an autoencoding neural network to extract relevant features and an artificial neural network to predict the quality (diameter and length of the workpiece). To apply a supervised learning method such as an artificial neural network, Wang et al. [3] assume that a small sample of sensor and actuator data is labeled manually. Therefore, the main contribution is the handling of small and imbalanced (only a few workpieces show quality defects) datasets. However, taking new samples and training new models, whenever a new product with new shape is produced, is highly labor- and time intensive. Unsupervised approaches need to be evaluated to reduce this effort.

Machine learning algorithms or, in general, the techniques of computational intelligence have the advantage that they do not require expert knowledge or the physical modeling of the process [4]. However, it should be considered to use the available, extensive knowledge of process experts, operators, and technicians for quality monitoring. Vogel-Heuser et al. [21] propose to combine expert knowledge and data mining in the form of cause-and-effect graphs. They compare the results of human mental models [22] with the results of regression algorithms based on process data. Further research considers algorithms such as Hebbian- and population-based algorithms in so-called fuzzy cognitive maps [23]. This expert knowledge can support data-driven quality systems-for example, in selecting the relevant parameters for model training. Even though expert knowledge acquisition and utilization is not a focus of this work, available knowledge will be used to evaluate the proposed method.

The discussed state of the art reveals extensive research in data-driven quality monitoring. However, the requirements for unsupervised quality monitoring in small data and changing environmental conditions demand the adaption and advanced development of existing algorithms to propose an efficient quality monitoring approach with high relevance for industrial applications.

\section{CONCEPT FOR PRODUCT QUALITY MONITORING IN HMPPS}

The identified challenges and the state of the art of data-driven quality monitoring specified the need for an unsupervised product quality monitoring approach for HMPP. In the following, a concept for such an approach is proposed. Two different algorithms-Dynamic Time Warping (DTW) and non-linear Random Forest Regression (RFR)-are evaluated to develop accurate and fast monitoring (cf. Sections 3.2 and 3.3). Both algorithms are applicable for unsupervised learning tasks, and the low complexity of the algorithms indicates the required flexibility to adjust the models to every single batch with different product parameters. Before the actual analysis is performed, the signals are preprocessed to isolate the essential phases of the process (cf. Section 3.1). The results of the product quality monitoring are visualized to enable the operator to assess the detected anomaly. This is repeated for each cycle with each workpiece monitored individually.

\subsection{Preprocessing: Signal Segmentation}

The process of powder compaction can be divided into different phases (cf. Section 1.1). The essential phases to detect cracks are depressurizing and demolding. Depressurizing describes the moment when the pressure is removed, and demolding is when the workpieces are ejected. The tensions between the workpiece and the mold can lead to cracks. Different approaches are considered to identify depressurizing and demolding automatically.

The terms signal segmentation and transient detection summarize algorithms and approaches to identify changes of a signal to segment phases with different characteristics. Constant 


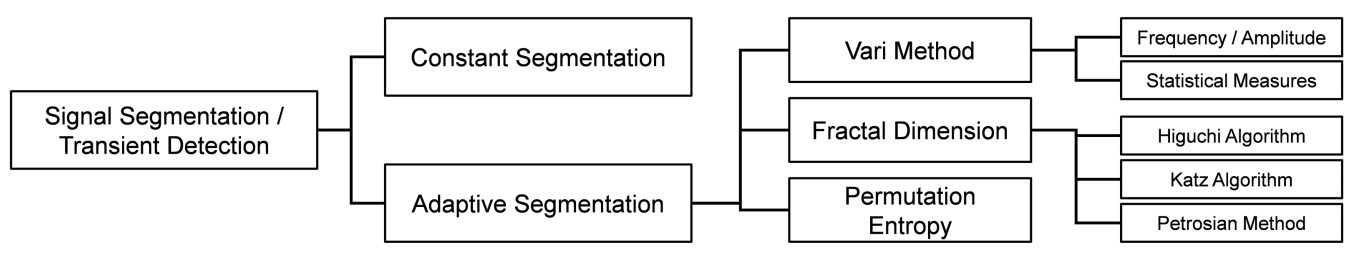

Fig. 2. Algorithms for signal segmentation/transient detection.

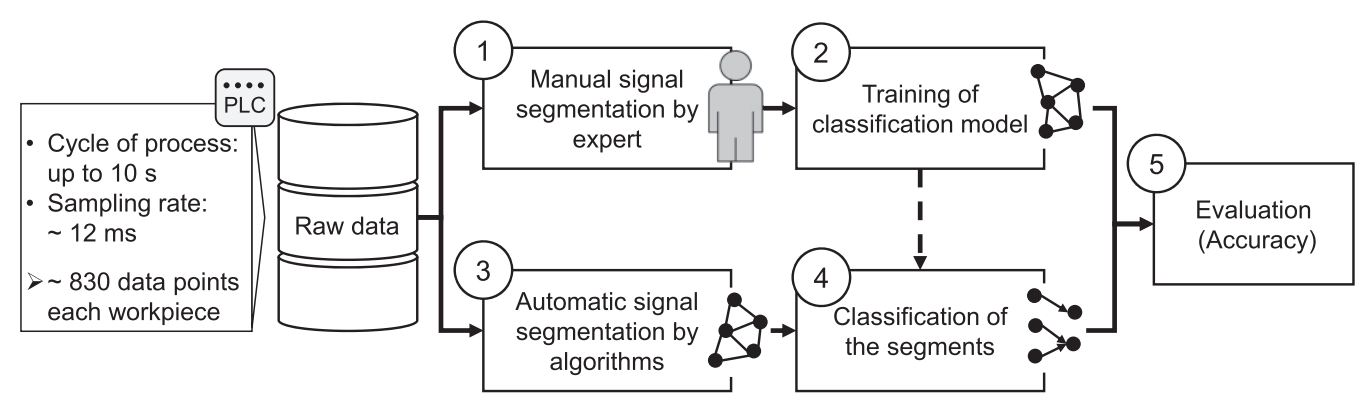

Fig. 3. Concept for the evaluation of a signal segmentation algorithm.

segmentation refers to a simple segmentation of the signal based on fixed window size, whereas adaptive segmentation allows a flexible segmentation based on changed behavior of the signal [24]. Different approaches are introduced for adaptive segmentation (cf. Figure 2). In the so-called Vari method, two sliding windows are combined to detect changes in the frequency or the amplitude of a signal [25]. Small differences between the two windows indicate a stable signal, whereas significant differences indicate changes in the behavior. This method can also be used with characteristics of the signal other than frequency or amplitude. Statistical measures such as standard deviation or slope are better suited for low-frequency data, which should be observed in the time domain rather than in the frequency domain. Fractal dimension is another concept, initially measuring the structure of an object. When applied to signals, the complexity of the signal is measured. The changes in the complexity indicate new phases. Several algorithms are introduced, calculating the fractal dimension: the Higuchi, Katz, and Petrosian algorithms [26]. These algorithms can be differentiated in their precision/accuracy and their sensitivity to noise. The Katz algorithm is most insensitive to noise; however, Higuchi is most accurate. A further measure for the complexity of a signal is the so-called permutation entropy [27]. In contrast to the classic Shannon entropy, the permutation entropy considers the temporal order of the values additionally and describes not only the distribution but also the complexity of the signal. Analogous to fractal dimension, the change of permutation entropy indicates a new phase due to a change of complexity.

To realize an automatic signal segmentation for the process of powder compaction, the mentioned algorithms are evaluated in Section 4.2. The results are compared in terms of their accuracy in detecting the phases. Experts of the process develop a dataset for evaluation by manually segmenting the signals (cf. Figure 3(1)), which are gathered from the programmable logic controller (PLC) of the HMPP. In this way, labeled data is produced, which is used to train a classification model (cf. Figure 3(2)). In parallel, the mentioned algorithms perform automatic segmentation of the signals (cf. Figure 3(3)). Based on the classification model, the detected segments are assigned to the phases (cf. Figure 3(4)). The comparison of the actual phase and the classified phase (cf. Figure 3(5)) will show the accuracy of the algorithms based on the confusion matrix. 


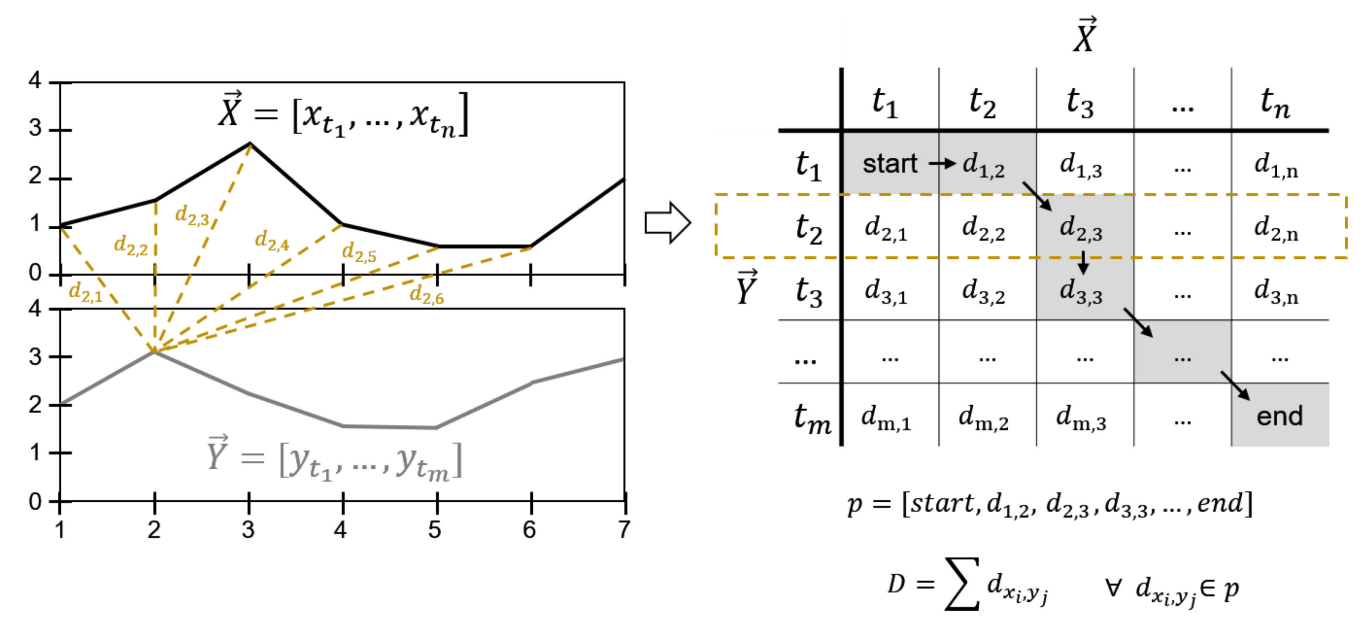

Fig. 4. Principle of the DTW algorithm.

\subsection{Dynamic Time Warping}

DTW was first introduced for speech recognition. Based on the similarity of the signal paths, DTW classifies speech to specific words with the most similar reference signal [28]. Beyond speech recognition, DTW indicates great potential for data mining in time series data, such as sensor data. DTW outperforms other similarity measures regarding its accuracy, particularly in small datasets [29]. For product quality monitoring, this algorithm can be used to compare the signal paths of the sensor data and to identify abnormal behavior leading to quality defects.

In the following, the DTW algorithm and its use for quality monitoring are explained briefly.

The DTW algorithm requires the input of two signals (1). It is not required that the two signals have the same length. This ensures the applicability to workpieces with different numbers of observations. The algorithm generates a matrix calculating the differences of the signal values for each point in time (2) to determine the similarity of the signals (cf. Figure 4).

$$
\begin{gathered}
\vec{X}=\left[x_{t_{1}}, \ldots, x_{t_{n}}\right], \vec{Y}=\left[y_{t_{1}}, \ldots, y_{t_{m}}\right] \\
d_{x_{i}, y_{j}}=\left|x_{i}-y_{j}\right| \forall i=\left[t_{1}, \ldots, t_{n}\right], j=\left[t_{1}, \ldots, t_{m}\right]
\end{gathered}
$$

The path $p$ with the minimal sum of distances through this matrix from position $(1,1)$ to $(m, n)$ corresponds to the distance of the two signals $D$. Thereby, the path needs to keep the following condition: the path can only proceed to the right, downward, or diagonally down to the right. The higher the distance of two signals $D$ is, the higher the differences between these signals are.

To detect an anomaly, a threshold for the distance measure $D$ has to be determined to differentiate normal distances of the signal paths and abnormal distances (cf. Figure 5(a)). It is the question when a distance of two signals exceeds the normal deviations. To derive a threshold, all distance measures of the pairwise calculations are considered jointly. A number of $N$ signals results in $N \cdot(N-1) / 2$ distance values. A simple outlier detection based on the concept of boxplots [30] is applied to detect abnormal distance measures (cf. Figure 5(b)). In this case, an outlier is a disproportionately high distance value. Normal distance values range between the first and the third quartile include $50 \%$ of the values. To identify disproportionately high values, this range of normal values (interquartile range IQR) is expanded by the factor 1.5 defining the threshold for outliers.

No model training is required for DTW, giving this method an advantage. Consequently, DTW is certainly applicable to small sample sizes. Furthermore, the algorithm considers the order of 
(a)

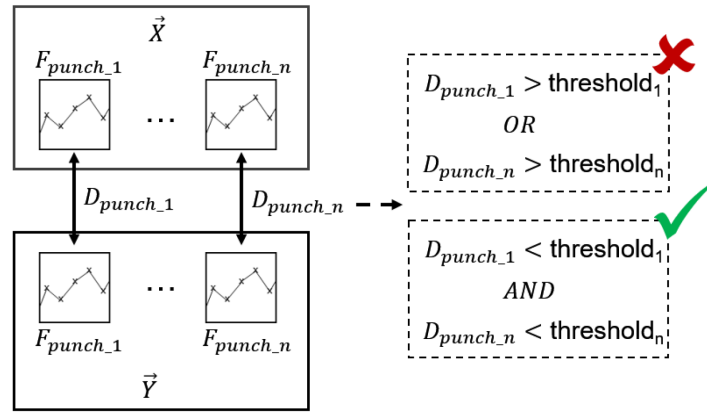

$D=$ Distance of two Signals based on Dynamic Time Warping

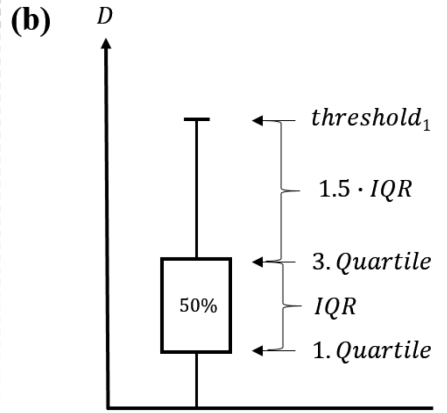

Fig. 5. Quality monitoring with DTW. (a) Concept of pairwise comparison of signals. (b) Determination of a threshold for abnormal distances.

the signals in time, detecting anomalies in the time structure. Regarding the time structure, delays are ignored, allowing the depressurizing and demolding phases to run slower or faster than expected without raising a false alarm. However, DTW considers each signal isolated regardless of the dependencies between the sensors and actuators, giving this method a disadvantage.

\subsection{Non-Linear RFR}

RFR is a powerful machine learning method for non-linear regression. In contrast to the wellknown support vector regression or neural networks, RFR does not require the determination of critical model parameters such as the kernel or the learning rate. In RFR, a large number of decision trees are built. Each of these trees provides one output to a specific input combination. Based on the mean of these outputs or the majority vote, the result is provided.

For product quality monitoring in HMPP, the input and output of an RFR model do not correspond to the sensor and the product quality data since no labeled data for product quality is given (unsupervised). The RFR model is trained to describe the dependencies between the position signals (input) and the force signals (output). For quality monitoring, the model output is compared to the actual force. If the deviation between the model and the actual value is significant, an anomaly will be detected (cf. Figure 6(a)). In this way, an unsupervised monitoring task is performed by a supervised learning method considering the dependencies of the sensor and actuator data of the HMPP. Anomalies are detected in the case that the signal values behave unexpectedly compared to the actuator data. Analogous to the concept of DTW, the threshold is defined based on the boxplot definition (cf. Figure 6(b)). A small dataset with samples is selected to train such an RFR model. The process experts need to ensure that these selected samples do not show any abnormal behavior. Otherwise, the model adapts to the abnormal behavior and is not able to detect irregularities in the depressurizing and demolding phases.

\section{IMPLEMENTATION OF THE PROPOSED APPROACH FOR PRODUCT QUALITY MONITORING}

The proposed approach is implemented in Python (Python 3.7) and evaluated based on a historic dataset. The following paragraphs discuss the available data and give an overview of the descriptive statistics. Furthermore, the proposed algorithms for signal segmentation and classification are evaluated. The results of the anomaly detection are presented and evaluated for DTW and RFR. 

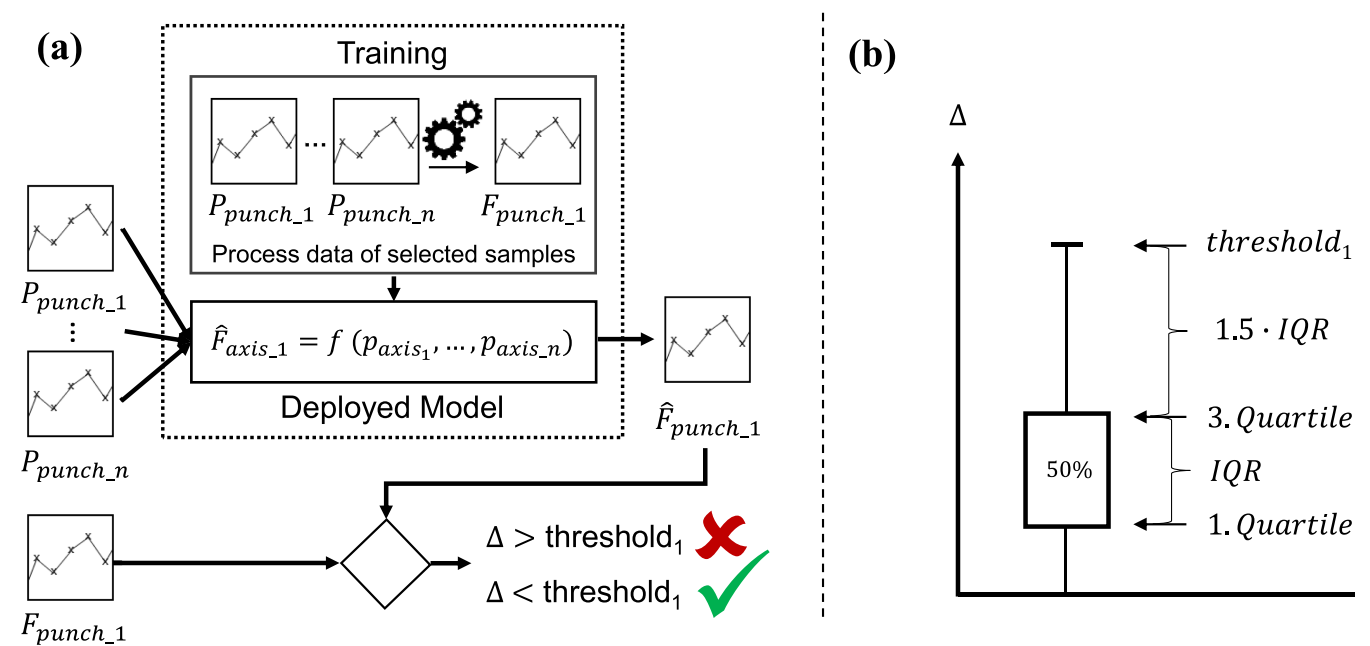

Fig. 6. Quality monitoring with RFR. (a) Concept for model training and model deployment. (b) Determination of a threshold for abnormal deviations.

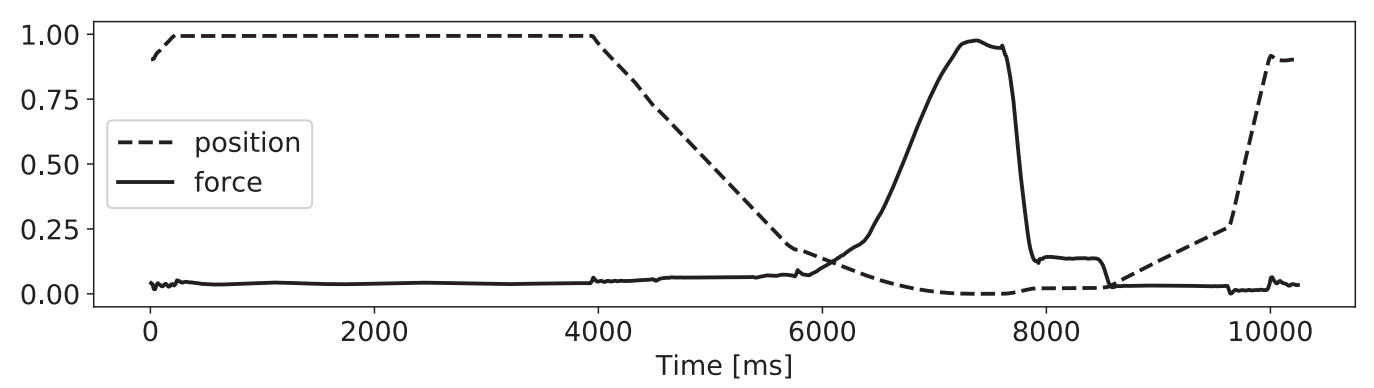

Fig. 7. Time diagram of position and force (normalized) of one exemplary punch of one workpiece.

\subsection{Descriptive Statistics of the Historic Dataset}

Test runs of 37 workpieces were performed, generating a labeled dataset for the evaluation of the proposed concept for a product quality monitoring approach for HMPP. This dataset links cracks in the workpiece to the anomalies and their locations in the process. Since it is required that the proposed approach achieves good results even on a small dataset, the evaluation of these test runs should demonstrate high accuracy in detecting anomalies despite the small number of samples. Anomalies, which were induced intentionally, occurred in two of the workpieces (5\%). The HMPP of the test runs is set up with four top punches and five bottom punches to produce the desired shape. In the following, the available dataset is further analyzed descriptively.

The production of one workpiece takes 10.23 seconds on average, with a standard deviation of $2.4 \mathrm{~ms}$. The small standard deviation shows that the process is very stable in time passing the different phases in almost identical routines. Since the sampling rate is $12 \mathrm{~ms}$, each workpiece consists of 853 data points resulting in a dataset with 31,561 data points in total. A position and force versus time diagram of one exemplary punch of one workpiece is shown in Figure 7 . The position of the punch is plotted as a dashed line, whereas the solid line depicts the force measured at this punch. In respect to secrecy and anonymization, the data is normalized between 0 and 1 . The process starts with almost 0 force and a high position of the punch. The drop of the punch (decreasing position) leads to an increased force signal reaching a maximum at around 7,200 ms 


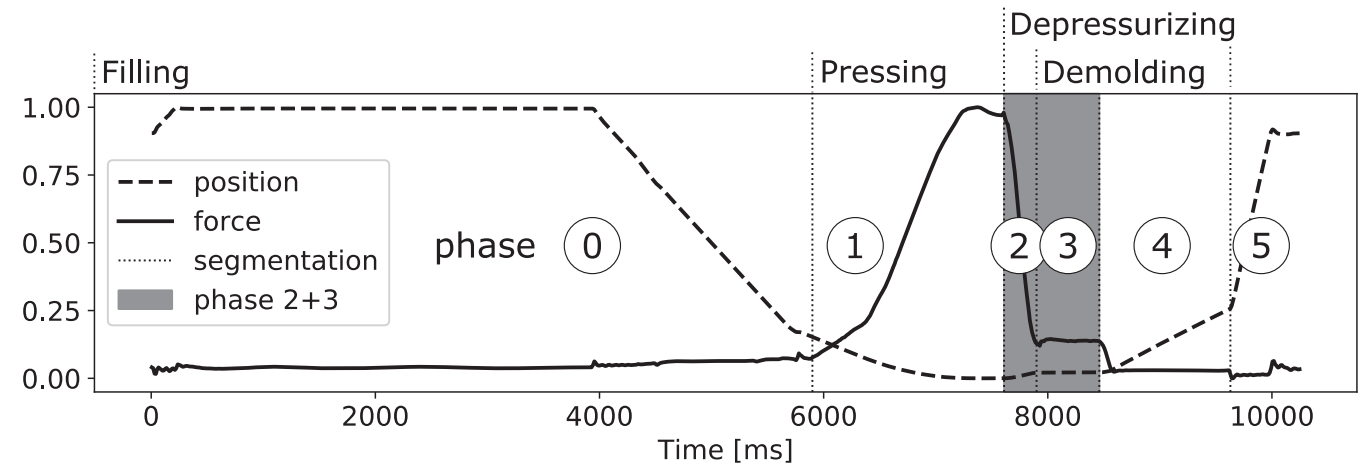

Fig. 8. Phases of the powder compaction process shown in the time diagram of the position and force (normalized) of the exemplary workpiece of Figure 7.

(pressing phase). The small increase in the position then leads to a drop in the force signal (depressurizing phase). During demolding, the force is kept at a small level and released totally through the increase of the position. Between the 37 workpieces of the test runs, only a small variance is detected, confirming the stability of the process.

\subsection{Signal Segmentation and Classification}

Different algorithms to segment the signals and isolate the relevant phases of the metal powder compaction process (cf. Section 3.1) are evaluated. As stated in Figure 3, manual segmentation is performed by experts of the process to achieve a data basis for comparison. The experts assess the phases of the process based on the characteristics of the actuator and sensor data. In particular, the position signal of one specific punch in combination with the corresponding force is the key indicator. A typical signal path for this data is already shown in Figure 7. The different phases of the process are added to the diagram in Figure 8. Furthermore, the relevant phases for quality monitoring are highlighted in light grey (phases 2 and 3). In contrast to the ASM handbook [2], this work splits the phase demolding into two several phases due to the experts' opinion.

Features such as minimum, maximum, mean, standard deviation, and slope are extracted from the different phases of the force and position signals to train a classification model based on the experts' segmentation. Furthermore, a moving window of 10 observations (i.e., data points) is applied to each phase extracting the mentioned features for smaller intervals. In this way, the characteristics of each phase are observable, increasing the validity of the classification model. Besides the data shown in Figure 8, the experts segmented further six workpieces of different shapes to increase the data basis for model training. The variation of the signal paths of these different workpieces is shown in Figure 9. The processing time of one workpiece differs widely between 3,000 and 7,000 $\mathrm{ms}$. Besides the processing times, the signal paths resemble one another and the different phases of the powder compaction process are clearly recognizable. This finding also emphasizes the choice of DTW. DTW observes the time behavior regardless of delays. Slower and faster processes can still be identified as identical or similar.

A decision tree is used to train this classification problem. The feature importance reveals that the slope of the force and the position signal, as well as the mean position, are the most important features for the classification task (Table 1). Phase 2 is characterized by a negative slope of the force signal, whereas the slope of the position is slightly positive and the mean position is rather low. Phase 3 is characterized by a slope of the force and position signal near zero and a rather low mean position. 
(a)

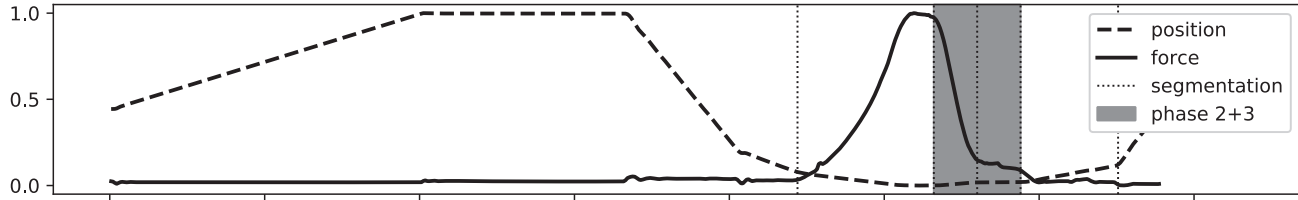

(b)

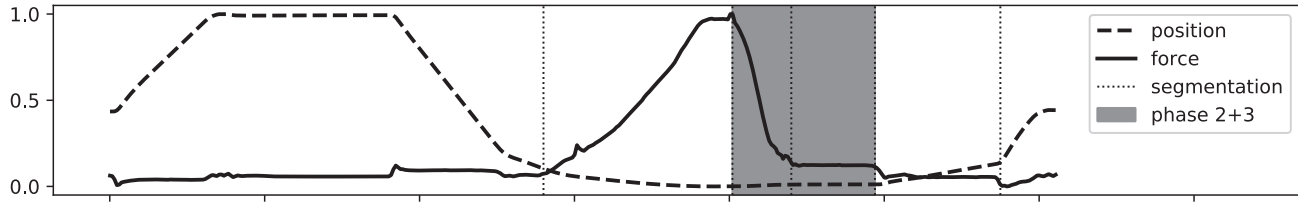

(c)

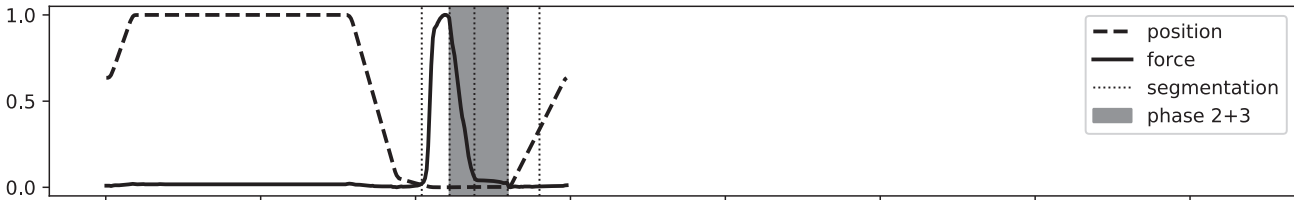

(d)

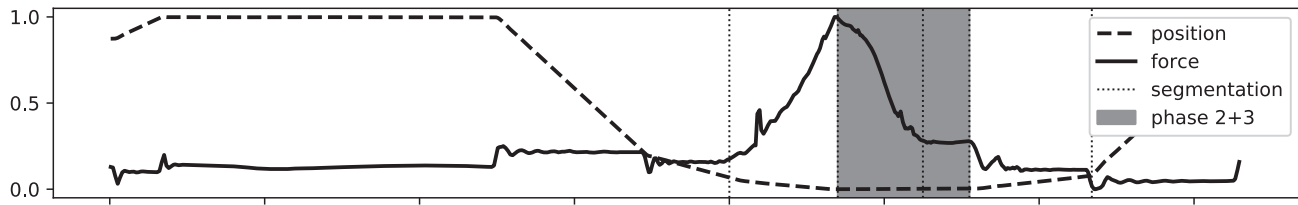

(e)

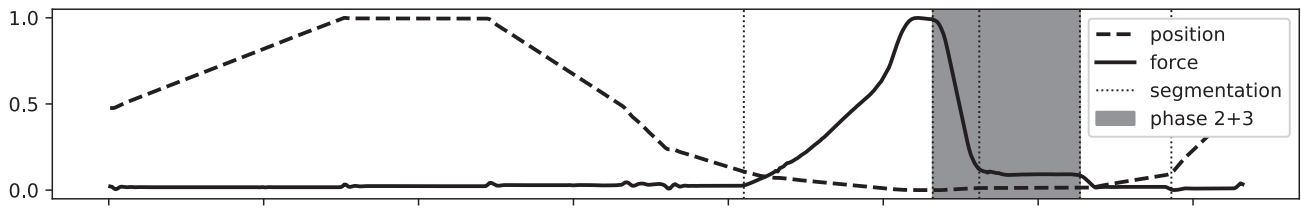

(f)

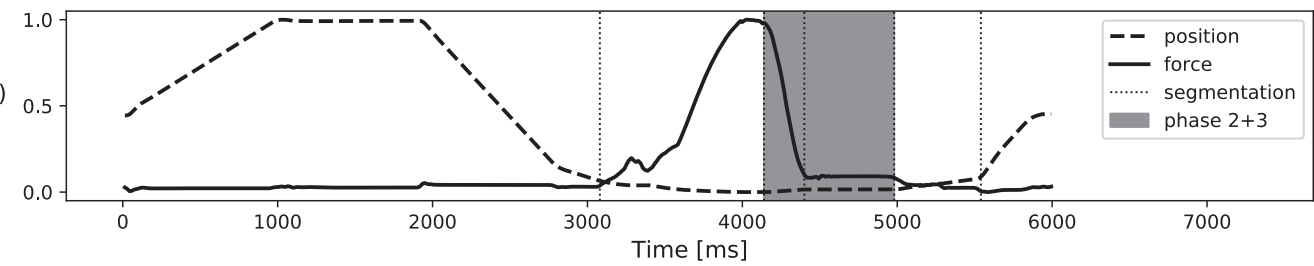

Fig. 9. Training data for classification. (a-f) Force and position signal for six workpieces with different shapes.

Table 1. Feature Importance of the Decision Tree for Classification of the Phases in the Powder Compaction Process

\begin{tabular}{lcccc}
\hline Features (Force) & Feature Importance & & Feature (Position) & Feature Importance \\
\cline { 1 - 1 } & 0.00597 & $\overline{\boldsymbol{P}}$ & 0.36100 \\
$F_{\text {min }}$ & 0.00000 & & $P_{\min }$ & 0.02780 \\
$F_{\text {max }}$ & 0.00522 & & $P_{\max }$ & 0.03388 \\
$F_{\text {std }}$ & 0.03097 & & $P_{\text {std }}$ & 0.02940 \\
$\overline{\boldsymbol{F}_{s}}$ & 0.23290 & & $\overline{\boldsymbol{P}_{s}}$ & 0.27286 \\
\hline
\end{tabular}

Note: The most important features are highlighted in bold font. 
In parallel to the training of the classification model, three different adaptive signal segmentation algorithms and a constant segmentation are applied to the force signal. The decision function of each algorithm $\left(G_{t}\right)$ is explained briefly in the following.

Vari method. Two moving windows describe the characteristics of a signal and thus identify changes that indicate a transition [25]. In this case, the slope $F s_{t}$ in a moving window of three observations describes the force signal. The difference of two successive moving windows $G_{t}^{\text {vari }}$ serves as basis for decision making.

$$
\begin{aligned}
F s_{t} & =\int_{t-3}^{t} F_{t} d t \\
G_{t}^{\text {vari }} & =F s_{t}-F s_{t-1}
\end{aligned}
$$

If the slope changes significantly, a transition will be detected. These significant changes are identified by the local maxima and minima of $G_{t}^{v a r i}$ (cf. dotted vertical lines in the second plot of Figure 10). A total of 28 segments are identified for the exemplary workpiece.

Fractal dimension. The Katz algorithm is applied to segment the force signal of the HMPP since this algorithm shows the best segmentation results in noisy data [26]. A moving window of 20 observations is set up to observe the fractal dimension over time $G_{t}^{\text {frac }}$ :

$$
G_{t}^{f r a c}=\left[\frac{\log _{10}(n)}{\log _{10}\left(\frac{d}{L}\right)+\log _{10}(n)}\right]_{t}^{t+20},
$$

where $L$ equals the sum of distances of $F_{t}, d$ equals the distance of the first observation of $F_{t}$ and the observation that is farthest away, and $n$ equals the number of observations. In other words, the spread of the signal within the window compared to the fluctuation characterize the window. If the fractional dimensions $G_{t}^{f r a c}$ reaches a local maximum, a transition will be detected (cf. dotted vertical lines in the third plot of Figure 10). A total of 17 segments are identified for the exemplary workpiece.

Permutation entropy. The permutation entropy describes the complexity of a signal by observing the probabilities of the sequential orders of successive points. Again, a moving window of 20 observations is set up to observe the permutation entropy over time $G_{t}^{e n t}$ :

$$
G_{t}^{e n t}=\left[-\sum_{j=1}^{n !} p_{j} \log _{2}\left(p_{j}\right)\right]_{t}^{t+20},
$$

where $p_{j}$ equals the probability of a specific sequence $j$. For signal segmentation, sequences of order 4 are considered [27]. If the complexity and the permutation entropy $G_{t}^{\text {ent }}$ respectively reach a local maximum, a transition of the signal will be detected (cf. dotted vertical lines in the fourth plot of Figure 10). A total of 20 segments are identified for the exemplary workpiece.

Besides the adaptive segmentation, a constant segmentation is applied for evaluation. Based on a fixed interval, the signal is segmented in equally sized sections. The length of the interval for the constant segmentation influences the results of the classification significantly. A smaller interval enables a finer segmentation, increasing the probability of catching the actual transitions. However, such small sections make it difficult to identify the real characteristics of the section, degrading the results of the classification. For this use case, a fixed interval of 15 observations achieved the best results (cf. dotted vertical lines in the fifth plot of Figure 10). A total of 56 segments are identified for the exemplary workpiece. 

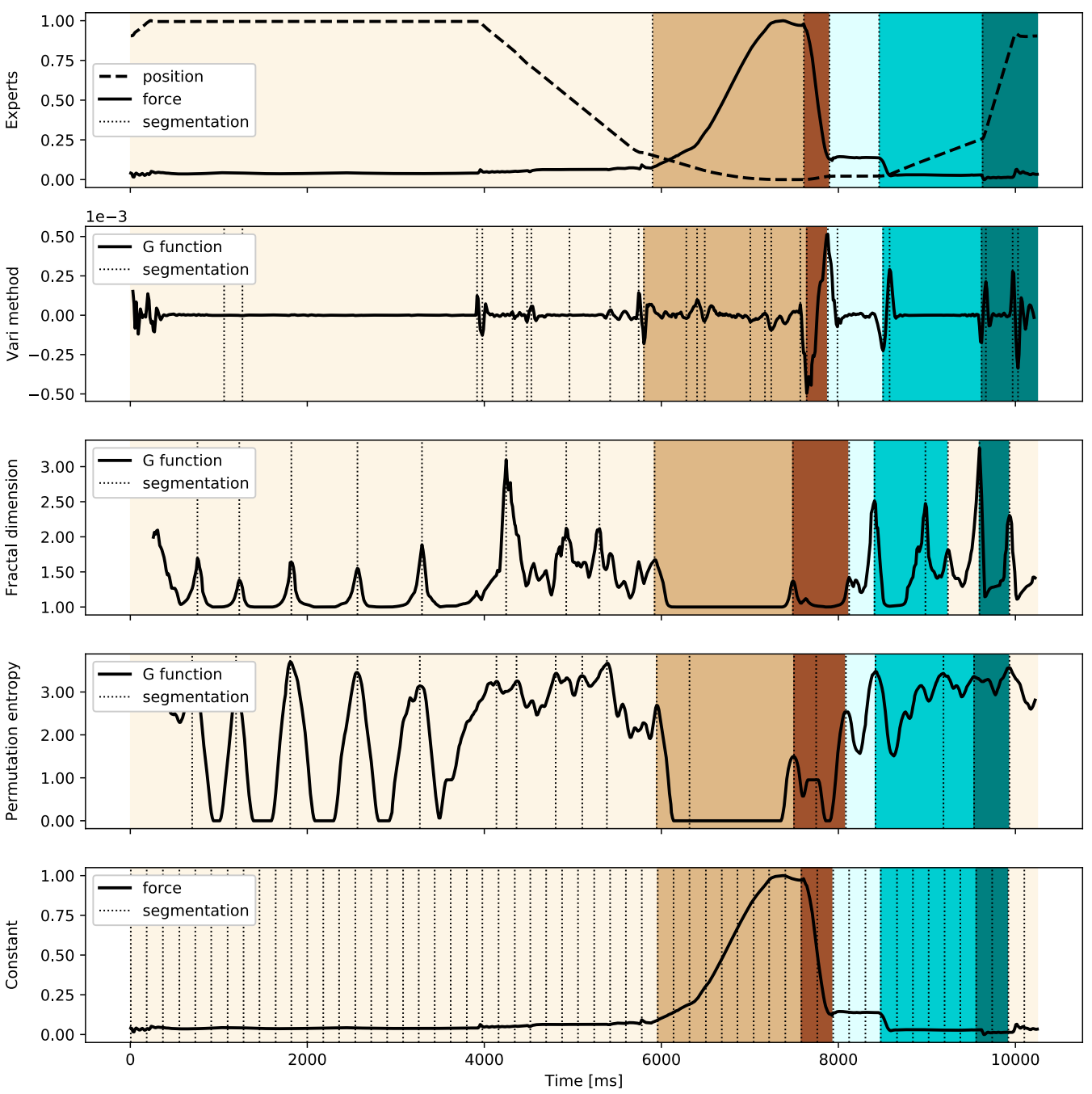

Fig. 10. Results of the adaptive and constant signal segmentation (dotted lines represent the segmentation of the signal) and the classification of the detected segments (colored areas reflect the assignment to a specific phase).

Based on the trained decision tree, the segments of the signals are classified. The color coding in Figure 10 reflects the results of this classification. The confusion matrices further show the accuracy of the different approaches (cf. Table 2). Overall, the accuracy of the Vari method is the highest (cf. marker 1 in Table 2). The results confirm that the method is accurate in classifying the phases in $97.42 \%$ of all observations. Minor time shifts are observable at the transitions resulting in the misclassification of observations to adjacent phases (cf. marker 2 in Table 2). In fractal dimension, the transitions of phase 2 are detected falsely (cf. markers 3 in Table 2). Furthermore, several segments of phases 4 and 5 are mixed up with phase 1 (cf. marker 4 in Table 2). Consequently, a high misclassification rate is observed. In permutation entropy, the accuracy reaches $92.15 \%$. The main reason for misclassification is again the falsely detected transitions of phase 2 and the mix-up of phases 5 and 1 (cf. markers 5 and 6 in Table 2). The constant segmentation shows rather good 
Table 2. Confusion Matrix for the Classification Results

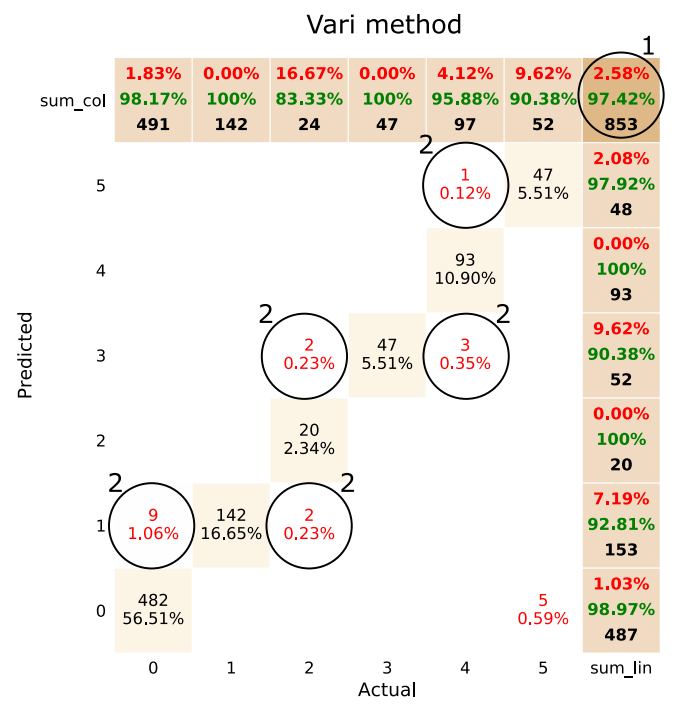

Permuation entropy

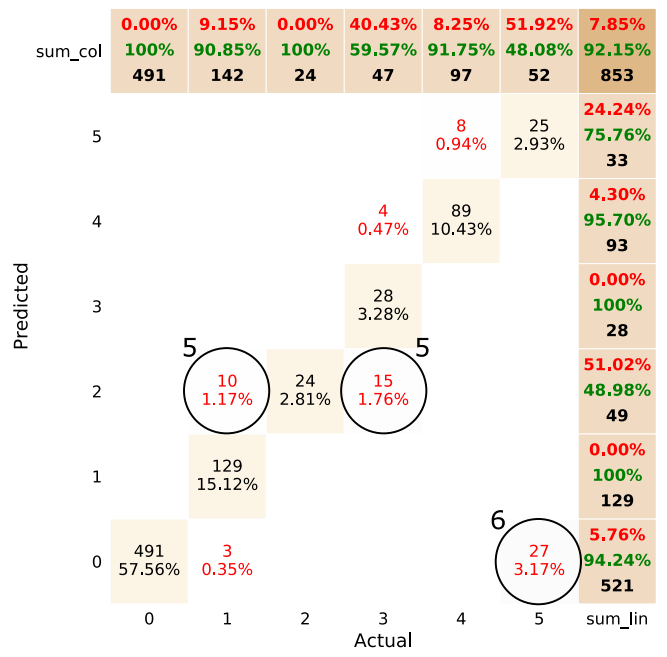

Fractal dimension

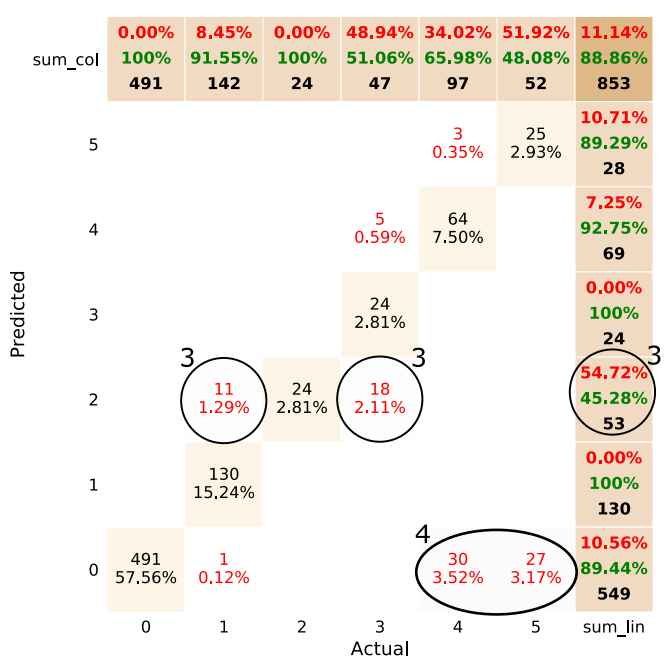

Constant segmentation

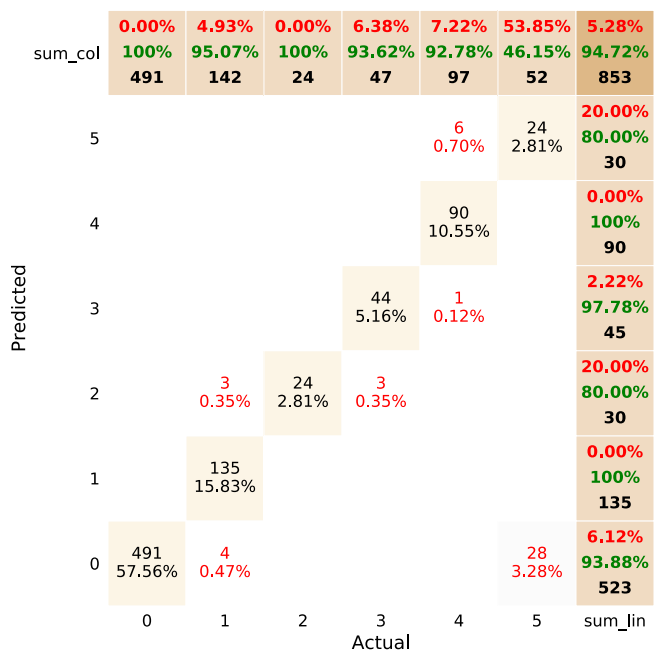

results in classification due to the incidentally suitable matches of the intervals and the transitions of the signal for this workpiece. However, the constant segmentation will not fit the faster process of, for example, workpiece C (cf. Figure 9). Since the Vari method achieves the best result, it is chosen for the automatic signal segmentation and classification.

\subsection{Anomaly Detection in Historic Data for Product Quality Monitoring}

Based on the segmented signal, DTW and RFR are applied to the phase of depressurizing and demolding.

4.3.1 Results of the DTW Approach. DTW is applied to each of the 37 workpieces and each of the nine punches separately. For each punch, $37 \cdot(37-1) / 2=666$ bilateral distance measures are calculated based on the DTW algorithm. The boxplots show the distribution of these bilateral 
(a)

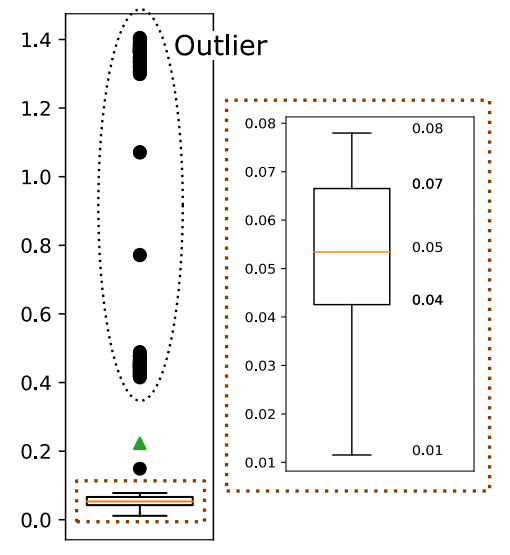

(b)

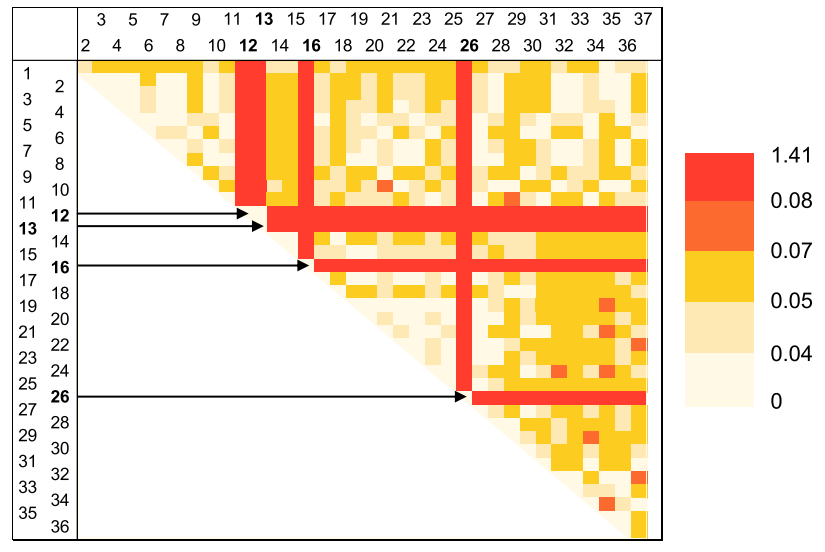

Fig. 11. Visualization of the results of DTW for punch 6. (a) Boxplot of the distances. (b) Heat map of the distance matrix.

Table 3. Variance and Threshold for Outliers of Each Punch

\begin{tabular}{lccccccccc}
\hline Punch & 1 & 2 & 3 & 4 & 5 & 6 & 7 & 8 & 9 \\
\hline Median & 0.069 & 0.044 & 0.005 & 0.074 & 0.163 & 0.053 & 0.137 & 0.038 & 0.435 \\
IQR & 0.031 & 0.034 & 0.004 & 0.251 & 0.103 & 0.024 & 0.303 & 0.053 & 0.292 \\
Threshold & 0.131 & 0.113 & 0.014 & 0.676 & 0.369 & 0.102 & 0.796 & 0.160 & 1.071 \\
\hline
\end{tabular}

distance measures for the punches separately. One example is given in Figure 11(a). Several outliers are detected exceeding the normal variations of the distance measures in punch 6 . The zoomed view shows that $50 \%$ of the distance measures range between 0.04 and 0.07 . Values such as 0.4 or higher are clearly out of this normal range. A heat map of the corresponding distance matrix for punch 6 is visualized in Figure 11(b). The outliers (distances bigger than 0.08) are marked in red, indicating abnormal behavior of the force signal path. The outliers are not distributed randomly around the matrix. Four specific workpieces (WP 12, 13, 16 and 26) show high distance measures to all others. The mean distance values for these workpieces are 1.295, 1.294, 0.466, and 0.465, respectively. Consequently, the DTW algorithm detects anomalies for these workpieces in the considered punch. The overview of the thresholds for outliers in all punches (Table 3) shows that normal variations in some punches are higher than in others. Since the data is normalized, they can be easily compared: punch 3 shows the lowest IQR (range, where $50 \%$ of the data is), and distances higher than 0.014 are already detected as outliers. In contrast, punch 7 shows the highest IQR, and the distance between the signal paths must be higher than 0.520 to be evaluated as an anomaly. Consequently, anomalies causing only small deviations are not easily detected in punch 7 since they become indistinct within the normal variations of this punch.

The results of the DTW distance measures for all punches are summarized in Table 4. Six workpieces indicate abnormal behavior in at least one punch. Since the anomaly detection reacts at the first sign (cf. Section 3.2), these six workpieces are rated as defective.

4.3.2 Result of the RFR Approach. To perform the non-linear regression for product quality monitoring, a model has to be trained on a subset of the data (cf. Section 3.3). For the test runs, a subset of 10 workpieces is used to train a prediction model for the force signals. These 10 workpieces are randomly chosen from the 35 workpieces without defects. The process is repeated 
Table 4. Final Results of the Anomaly Detection Using DTW

\begin{tabular}{lllllllll}
\hline Punch & 1 & 2 & 3 & 4 & 5 & 6 & 7 & 8 \\
\hline WP 4 & & & & & & & & $\times$ \\
WP 9 & & & & & & & & $\times$ \\
WP 12 & & & & & & $\times$ & & $\times$ \\
WP 13 & & & & & $\times$ & & $\times$ \\
WP 16 & $\times$ & & & & & $\times$ & & \\
WP 26 & & & & & $\times$ & $\times$ & \\
{$[\ldots]$} & & & & & & & \\
\hline
\end{tabular}

Note: Abnormal behavior of workpieces is marked with an $\mathrm{X}$ for the corresponding punch.

Table 5. Coefficient of Determination for the Regression Models and the Determined Threshold for Anomalies for Each Punch

\begin{tabular}{lccccccccc}
\hline Punch & 1 & 2 & 3 & 4 & 5 & 6 & 7 & 8 & 9 \\
\hline mean $\left(R^{2}\right)$ & 0.999 & 0.996 & 0.971 & 0.996 & 0.997 & 0.994 & 0.947 & 0.990 & 0.874 \\
std $\left(R^{2}\right)$ & 0.000 & 0.001 & 0.010 & 0.002 & 0.002 & 0.08 & 0.060 & 0.008 & 0.042 \\
Threshold & 0.0112 & 0.0124 & 0.0021 & 0.0325 & 0.0144 & 0.0128 & & 0.0197 & \\
\hline
\end{tabular}

10 times to perform cross validation. The result of each iteration (coefficient of determination $R^{2}$ ) is observed. In this way, the validity of the models is evaluated. A high mean coefficient of determination confirms that the model can cover the dependencies of the system and match changes of the input to the resulting output. A small standard deviation of this coefficient across the 10 iterations shows that the results are independent of the choice of the training subset. The mean coefficient of determination and the standard deviation demonstrate a high validity of the models except for punches 7 and 9 (cf. Table 5). In contrast to the other punches, punches 7 and 9 show a lower mean coefficient of determination and a higher standard deviation. This shows that the model cannot represent the behavior entirely and that the model is dependent on the randomly chosen subset, which is used for model training. Consequently, these models cannot be used for anomaly detection.

Based on the remaining punches, anomaly detection is performed for the workpieces that are not part of the model training. The predicted force is compared to the actual force for each punch and workpiece. The so-called residual (the difference between predicted and actual value) is used to determine abnormal behavior.

Analogous to DTW, a boxplot of the residuals determines the thresholds (cf. Table 5). If a punch shows successive points, where the difference between predicted and actual value exceeds the threshold, an anomaly will be detected. Single points exceeding the threshold are seen as exceptions, which are not leading to anomalies.

For each iteration, workpieces with anomalies in at least one punch are extracted. To summarize the results, the anomalies across the iterations are counted for each workpiece separately. Furthermore, it is observed if the specific workpieces have been part of the randomly chosen training data subset. Finally, three workpieces show anomalies in at least one punch in every single iteration, where the workpiece does not belong to the training data subset (Table 6). Furthermore, six workpieces show anomalies in at least eight iterations, indicating an abnormal behavior. Finally, nine workpieces are rated as abnormal, demanding a quality inspection. 
Table 6. Results After 10 Iterations of the Regression Model

\begin{tabular}{lccccccccc|ccc}
\hline WP & 16 & 26 & 1 & 4 & 25 & 12 & 11 & 28 & 29 & 13 & 9 & {$[\ldots]$} \\
\hline Anomaly & 10 & 10 & 8 & 8 & 8 & 7 & 5 & 5 & 5 & 7 & 6 & \\
WP in train data & 0 & 0 & 2 & 1 & 0 & 1 & 3 & 3 & 3 & 0 & 1 & \\
Sum & 10 & 10 & 10 & 9 & 8 & 8 & 8 & 8 & 8 & 7 & 7 & \\
\hline
\end{tabular}

Table 7. Confusion Matrix for DTW and RFR

\begin{tabular}{lc|cc|c}
\hline & & \multicolumn{2}{|c|}{ Actual } & \\
& & Positive & Negative & Sum \\
\hline Predicted & Positive & $2 / 2$ & $4 / 7$ & $6 / 9$ \\
DTW/RFR & Negative & $0 / 0$ & $31 / 28$ & $31 / 28$ \\
\hline & Sum & $2 / 2$ & $35 / 35$ & $37 / 37$ \\
\hline
\end{tabular}

4.3.3 Evaluation of the Anomaly Detection Approach for Product Quality Monitoring. DTW detects six anomalies, whereas RFR finds nine anomalies, which should be examined in quality inspections. The confusion matrix in Table 7 shows the accuracy of both approaches.

DTW and RFR detect both induced anomalies (WP16 and 26) correctly. This results in a truepositive rate of $100 \%$ ( 2 out of 2). False-discovery rates of $66 \%$ ( 4 out of 6 ) and $77 \%$ ( 7 out of 9) indicate a high number of false alarms: the quality monitoring approach detects an anomaly, whereas the workpiece shows no defects. Two of the false positives in DTW and RFR are common (WP4 and 12). A detailed look at the force and the predicted force for WP12 in punch 6 is given in Figure 12(a). Furthermore, the force of WP20 with normal behavior is given as a basis for comparison. WP20 and WP12 show a different signal path in the considered phase, resulting in high distance values for DTW. The predicted force signal of RFR differs significantly from the actual force signal, leading to an anomaly alarm also in RFR. However, this unexpected behavior did not lead to cracks and the alarm must be ranked as a false positive even though an apparent deviation is observable. To give a true-positive example, the force signal path of WP16 is given for punch 1 in Figure 12(b).

A visualization embedded in a user interface should be developed to enable the operator of the HMPP to assess the anomalies. An operator with extensive knowledge of the machine and process may evaluate the detected anomalies to identify and isolate defective workpieces. Therefore, the force signal of a detected workpiece is displayed to the operator. As seen in Figure 12, the expected force signal, as well as the force signal of a high-quality workpiece, are displayed in parallel to enable the operator to compare. The interval with the anomaly is highlighted. In this way, the operator may evaluate the anomalies based on the location, shape, and length. If an anomaly alarm such as in Figure 12(a) shows harmless deviations from the expected signal path, the operator will evaluate it as such and avoid unnecessary quality inspections of this workpiece. This requires a profound knowledge of the dependencies and behavior of the HMPP, which is assumed for experienced operators. Inexperienced operators may have difficulties identifying false alarms leading to a higher number of required quality inspections. However, these false positives are acceptable. Due to high warranty costs, the true-positive rate is given more importance.

The evaluation of such a user interface in an industrial application is still pending.

4.3.4 Computation Time. The analysis is implemented in Python (Python 3.7). The algorithms are executed on Windows Server 2016 machine virtualized via Hyper-V. The host computer contains an Intel Xeon E5-2690 v4 CPU with a total of 28 physical cores at $2.6 \mathrm{GHz}$ with HyperThreading enabled. Moreover, 128 GB of RAM are available. The virtual machine has full and 


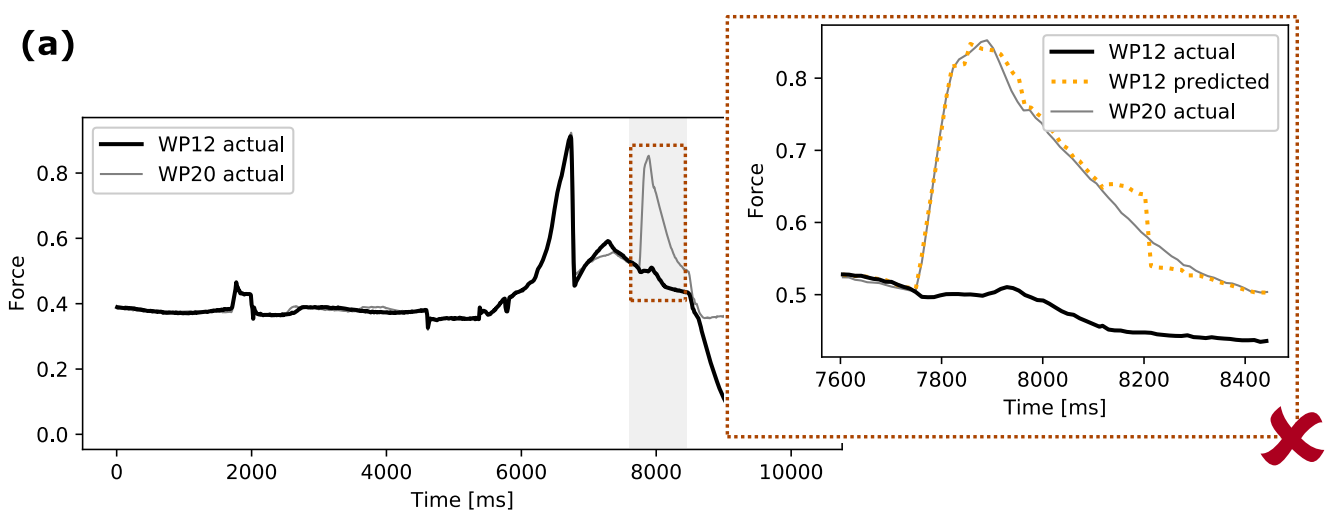

\section{(b)}

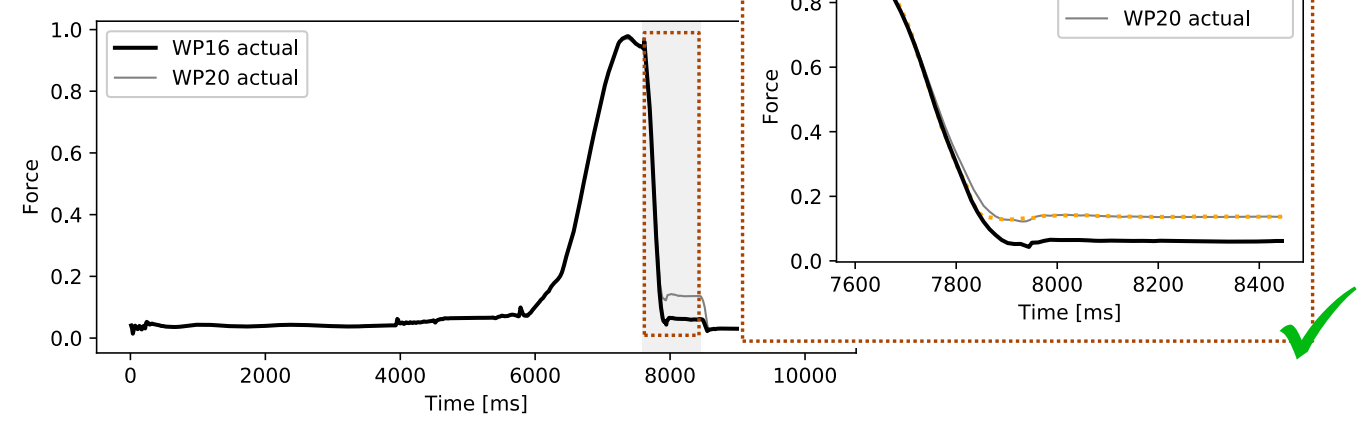

Fig. 12. Visual representation of the results of the quality monitoring approach for two selected workpieces. (a) Wrongly detected anomaly of WP12 in punch 6. (b) Correctly detected anomaly of WP16 in punch 1.

prioritized access to the host resources. All measures are based on these conditions. The algorithms are performed nine times-once for each punch-providing the data base for the following evaluation.

For DTW, the Python package "dtaidistance" version 1.2.3 is used. Mostly, simple calculations, particularly subtraction, are performed. Searching the shortest path through the matrix (cf. Figure 4 ) is the most complex arithmetic operation. Since the signals of the considered workpiece contain 853 data points each, an $853 \times 853$ matrix is formed. On average, the calculation of the DTW distance between two signals of this length takes 0.0222 seconds. Since the considered use case includes $z=9$ different punches, the comparison of two workpieces takes on average $9 \cdot 0.0222 \approx 0.2$ seconds. Based on $w=37$ workpieces and $(37 \cdot(37-1) / 2)=666$ bilateral calculations, a computation time of $666 \cdot 0.2=133.2$ seconds is achieved for the calculation of the DTW distances in the test run. However, for the online operation of the approach, it is only required to calculate the new distance measure between the new workpiece and all former workpieces. Therefore, the computational time is reduced to $w \cdot z \cdot 0.0222$ seconds for each new workpiece.

For RFR, the "RandomForestRegressor" of the Python package "scikit-learn" version 0.22 .111 is used. The computational complexity consists of two aspects. First, an RFR model is trained predicting the force signals based on the input signals and the trained model. Second, the calculation of the deviations of predicted and actual force values is performed. The training of an RFR model based on a training dataset with 10 workpieces takes on average 0.3062 seconds. Since nine punches are 
considered in this use case, the computational time of $9 \cdot 0.3062 \approx 2.76$ seconds is achieved. At first glance, this seems to be critical for a fast product quality monitoring, but this time is only required once for training. Based on the trained model, the detection of anomalies takes on average 0.0126 seconds for each punch. Consequently, the use case with nine punches achieves the computation time of $9 \cdot 0.0126 \approx 0.11$ seconds for each workpiece.

In total, the proposed product quality monitoring approach performs the anomaly detection in only a few seconds, ensuring that the result is available before the next workpiece is produced by the HMPP. Although DTW has a higher computation time for each workpiece since bilateral distance measures need to be calculated, RFR has a high initial computation time but performs the anomaly detection in around 0.11 seconds each workpiece. Possible data preprocessing steps as data cleaning or others are not considered here. If required, they would slow down the analysis. However, the implementation is not optimized in terms of parallelization yet. Possible savings in computational time still need to be elaborated for this implementation.

4.3.5 Discussion. The proposed approach for product quality monitoring shows consistent results in the industrial application of an HMPP. The algorithms DTW and RFR perform the unsupervised learning task (requirement 1) for product quality monitoring reliably. DTW can be applied already to two workpieces, whereas RFR requires a small training dataset. Still, the concept allows for a quick adaption to new products with different shapes (requirement 3) since already minimal sample sizes are sufficient for model training. Further, the visualization of the anomaly (cf. Figure 12) enables the operator of the assessment based on the location, shape, and length of the anomaly (requirement 2). The evaluation shows that the proposed methods achieve a true-positive rate of $100 \%$. However, a high number of false alarms is observed, revealing drawbacks that must be addressed for industrial applications. Comparing DTW and RFR, the following must be considered: DTW does not require a training dataset and is already applicable for a small number of workpieces. However, DTW considers each signal path isolated, regardless of the dependencies between the signals. A single signal path may occur abnormally while observing stable interactions of the punches. In this case, DTW will detect an anomaly regardless of the normal operations. A regression model, which covers the dependencies between the punches, is demanded to complement the results of DTW. However, RFR and regressions in general show some drawbacks. A dataset containing data of normal operation is required for model training. Consequently, the results of the regression are not available from the beginning of a batch, as is possible for DTW. The regression benefits from a sound dataset, suggesting a late activation of the anomaly detection. Since the methods can compensate their drawbacks, a combination of both DTW and RFR is proposed. For the application in production environments, continuous retraining of the regression model is proposed, refining the results. Furthermore, the thresholds for the detection of anomalies must be adapted to the feedback to receive a continuously learning system. Although the detection approach is more sensitive in the beginning, the detection of many anomalies will make the approach more robust over time. The approach adapts the thresholds based on feedback if a detected anomaly has led to a crack or not, decreasing the rate of false alarms.

\section{CONCLUSION AND OUTLOOK}

Product quality monitoring is one of the promising applications available for machine learning and artificial intelligence in CPS. It is of interest particularly in cases where the quality parameters cannot be measured with reasonable effort, such as in the considered use case of powder compaction in HMPPs. However, challenges such as a very small sample size of only 37 workpieces and a fast process of 10 seconds set high requirements for data-driven product quality monitoring. Therefore, this article introduces an approach using DTW and RFR to realize an unsupervised 
anomaly detection for unlabeled data of an HMPP. This data consists of force and position signals of the punches in an HMPP, recorded at a sampling rate of approximately $12 \mathrm{~ms}$. The approach aims at comparing the signal paths of the relevant parameters between different workpieces rather than modeling the complex inner physical processes of powder compaction. This allows a fast adaptation of the approach to different batches with changing product parameters such as shape. Furthermore, expensive retrofitting of sensors, measuring the physical conditions of the process in more detail, is superfluous. The evaluation shows that a small sample size of 37 workpieces is sufficient to train models, define thresholds, and perform anomaly detection. Prior to the anomaly detection, algorithms for signal segmentation are introduced in this work to focus the detection on the distinct and most relevant phases of the process. For the considered HMPP, a classification model based on the decision tree algorithm is trained to data, which is labeled manually by experts. The evaluation based on an industrial dataset from test runs demonstrates the accuracy of the signal segmentation approach: $97.42 \%$ of the data points are correctly classified to their corresponding process phase. The evaluation of the proposed anomaly detection based on DTW and RFR shows a true-positive rate of $100 \%$ while preserving an acceptable false-positive rate (hypothesis 1). The approach also ensures that the result is available within 10 seconds before the next workpiece is finished (hypothesis 2). In this way, efficient quality management is introduced for fast processes with minimal sample sizes requiring time- and cost-intensive quality inspections for single workpieces only if anomalies are detected in the production process. The proposed approach is also applicable for other types of presses or processes with a limited number of relevant parameters and a specific and strict procedure.

In future work, the proposed approach should be implemented as a self-learning system adapting models and thresholds dynamically based on feedback. Therefore, the algorithms and software must be wrapped in autonomous agents, interacting with their environment. Retraining models, as well as the adaption of the thresholds for the anomaly detection, should be triggered by the feedback of quality inspections. If a high-quality workpiece has been detected as abnormal, the data of this workpiece is used to supplement the training dataset and to adjust the thresholds to avoid comparable false alarms in the future. The socio-technical system should be considered in addition to the technical extension of the approach. The working environment needs to support the operator of the HMPP in using such a quality monitoring approach and in giving feedback for improvement. In addition to the implementation in production environments, a refinement of the determination of outliers indicating abnormal behavior should be considered. Apart from the distance measure in DTW and the residual in RFR, further features could be used to specify anomalies. One feature could be the time span of how long the deviations last. Compared to the long-lasting deviations, it could be of interest if a small deviation is present permanently or a short and significant deviation is observed. These features could be used to train a classification model for anomaly detection to decrease false alarms. Finally, another promising approach could be to examine, augment, and support the proposed methodology by additional data such as information about alarms, tooling, setup, and the like.

\section{ACKNOWLEDGMENTS}

The Institute of Automation and Information Systems thanks its industrial partners for supporting this research.

\section{REFERENCES}

[1] B. Vogel-Heuser and D. Hess. 2016. Guest editorial industry 4.0-Prerequisites and visions. IEEE Transactions on Automation Science and Engineering 13, 2 (2016), 411-413. DOI : 10.1109/TASE.2016.2523639 
[2] ASM International. 2002. Powder Metal Technologies and Applications (10th ed). ASM International, Materials Park, $\mathrm{OH}$.

[3] G. Wang, A. Ledwoch, R. M. Hasani, R. Grosu, and A. Brintrup. 2019. A generative neural network model for the quality prediction of work in progress products. Applied Soft Computing 85 (2019), 106683. DOI : 10.1016/j.asoc.2019. 105683

[4] R. D. Labati, A. Genovese, E. Muñoz, V. Piuri, and F. Scotti. 2018. Applications of computational intelligence in industrial and environmental scenarios. In Learning Systems: From Theory to Practice. Studies in Computational Intelligence, Vol. 756. Springer, 29-46.

[5] I. Kirchen, B. Vogel-Heuser, O. Hildenbrand, R. Schulte, M. Vogel, M. Lechner, and M. Merklein. 2017. Data-driven model development for quality prediction in forming technology. In Proceedings of the 2017 IEEE 15th International Conference on Industrial Informatics (INDIN'17). 775-780. DOI : 10.1109/INDIN.2017.8104871

[6] L. Yao and Z. Ge. 2019. Nonlinear Gaussian mixture regression for multimode quality prediction with partially labeled data. IEEE Transactions on Industrial Informatics 15, 7 (2019), 4044-4053. DOI : 10.1109/TII.2018.2885363

[7] X. Li, F. Wu, R. Zhang, and F. Gao. 2019. Nonlinear multivariate quality prediction based on OSC-SVM-PLS. Industrial \& Engineering Chemistry Research 58, 19 (2019), 8154-8161. DOI : 10.1021/acs.iecr.8b06079

[8] S. Lalam, P. K. Tiwari, S. Sahoo, and A. K. Dalal. 2019. Online prediction and monitoring of mechanical properties of industrial galvanised steel coils using neural networks. Ironmaking \& Steelmaking 46, 1 (2019), 89-96. D0I : 10.1080/ 03019233.2017.1342424

[9] C. Gonzalez-Val, A. Pallas, V. Panadeiro, and A. Rodriguez. 2020. A convolutional approach to quality monitoring for laser manufacturing. Fournal of Intelligent Manufacturing 31, 3 (2020), 789-795. DOI : 10.1007/s10845-019-01495-8

[10] S. A. Shevchik, T. L. Quang, F. V. Farahani, F. Neige, B. Meylan, S. Zanoli, and K. Wasmer. 2019. Laser welding quality monitoring via graph support vector machine with data adaptive kernel. IEEE Access 7 (2019), 93108-93122. DOI : 10.1109/ACCESS.2019.2927661

[11] Y. Oh, M. Busogi, K. Ransikarbum, D. Shin, D. Kwon, and N. Kim. 2019. Real-time quality monitoring and control system using an integrated cost effective support vector machine. fournal of Mechanical Science and Technology 33 , 12 (2019), 6009-6020. DOI : 10.1007/s12206-019-1145-9

[12] J. P. Liu, O. F. Beyca, P. K. Rao, Z. J. Kong, and S. T. S. Bukkapatnam. 2017. Dirichlet process Gaussian mixture models for real-time monitoring and their application to chemical mechanical planarization. IEEE Transactions on Automation Science and Engineering 14, 1 (2017), 208-221. DOI : 10.1109/TASE.2016.2599436

[13] Y. Tan, C. Niu, H. Tian, L. Hou, and J. Zhang. 2019. A one-class SVM based approach for condition-based maintenance of a naval propulsion plant with limited labeled data. Ocean Engineering 193 (2019), 106592. DOI : 10.1016/j.oceaneng. 2019.106592.

[14] Y. Xiao, H. Wang, W. Xu, and J. Zhou. 2016. Robust one-class SVM for fault detection. Chemometrics and Intelligent Laboratory Systems 151 (2016), 15-25. DOI : 10.1016/j.chemolab.2015.11.010

[15] H. Y. Yu, J. L. Tang, and Y. Tang. 2015. Research on $800 \mathrm{MN}$ hydraulic press monitoring system. Materials Research Innovations 19, S6 (2015), S6-181-S6-183. DOI : 10.1179/1432891715Z.0000000001474

[16] H. Chen, J. P. Tan, J. L. Gong, Z. Q. Shu, X. Y. Cao, and J. N. Zhou. 2011. Design of new condition monitoring system and data analysis in large forging die hydraulic press. Advanced Materials Research 311-313 (2011), 1546-1550. DOI : 10. 4028/www.scientific.net/AMR.311-313.1546

[17] D. Sowmiya. 2013. Monitoring and control of a PLC based VFD fed three phase induction motor for powder compacting press machine. In Proceedings of the 2013 7th International Conference on Intelligent Systems and Control (ISCO'13). $90-92$.

[18] J. A. Ferreira, P. Sun, and J. J. Gracio. 2006. Design and control of a hydraulic press. In Proceedings of the IEEE International Symposium on Computer-Aided Control Systems Design. 814-819.

[19] M. I. Rîpanu, G. Nagîț, V. Merticaru, M. A. Mihalache, M. Boca, and V. Huşanu. 2014. An optimized methodology for process quality analysis and monitoring activities in case of sheet metal bearing cages stamping. Applied Mechanics and Materials 657 (2014), 183-187. DOI : 10.4028/www.scientific.net/AMM.657.183

[20] H. Du, B. Huang, L. Wang, and S. M. Chen. 2014. The design of monitoring system in large hydraulic press aiming at the precise closed-loop control. Applied Mechanics and Materials 989-994 (2014), 3062-3069. DOI: 10.4028/www. scientific.net/AMR.989-994.3062

[21] B. Vogel-Heuser, V. Karaseva, J. Folmer, and I. Kirchen. 2017. Operator knowledge inclusion in data-mining approaches for product quality assurance using cause-effect graphs. In Proceedings of the 20th IFAC World Congress. 1358-1365. DOI : $10.1016 /$ j.ifacol.2017.08.233

[22] D. Pantförder, J. Schaupp, and B. Vogel-Heuser. 2017. Making implicit knowledge explicit-Acquisition of plant staff's mental models as a basis for developing a decision support system. In Proceedings of HCI International 2017: Posters Extended Abstracts. 358-365. 
[23] E. I. Papageorgiou. 2012. Learning algorithms for fuzzy cognitive maps-A review study. IEEE Transactions on Systems, Man, and Cybernetics: Systems 42, 2 (2012), 150-163. DOI : 10.1109/TSMCC.2011.2138694

[24] M. Anisheh and H. Hassanpour. 2009. Adaptive segmentation with optimal window length scheme using fractal dimension and wavelet transform. International fournal of Engineering 22, 3 (2009), 257-268. http://www.ije.ir/article_ 71799.html.

[25] H. Azami, K. Mohammadi, and B. Bozorgtabar. 2012. An improved signal segmentation using moving average and Savitzky-Golay filter. Journal of Signal and Information Processing 3, 1 (2012), 39-44. DOI : 10.4236/jsip.2012.31006

[26] R. Esteller, G. Vachtsevanos, J. Echauz, and B. Litt. 2001. A comparison of waveform fractal dimension algorithms. IEEE Transactions on Circuits and Systems I 48, 2 (2001), 177-183. DOI : 10.1109/81.904882

[27] M. Riedl, A. Müller, and N. Wessel. 2013. Practical considerations of permutation entropy. European Physical fournal Special Topics 222, 2 (2013), 249-262. DOI : 10.1140/epjst/e2013-01862-7

[28] H. Sakoe and S. Chiba. 1978. Dynamic programming algorithm optimization for spoken word recognition. IEEE Transactions on Acoustics, Speech, and Signal Processing 26, 1 (1978), 43-49. DOI : 10.1109/TASSP.1978.1163055

[29] H. Ding, G. Trajcevski, P. Scheuermann, X. Wang, and E. Keogh. 2008. Querying and mining of time series data. Proceedings of the VLDB Endowment 1, 2 (2008), 1542-1552.

[30] R. L. Nuzzo. 2016. The box plots alternative for visualizing quantitative data. PM \& R: The fournal of Injury, Function, and Rehabilitation 8, 3 (2016), 268-272. DOI : 10.1016/j.pmrj.2016.02.001

Received May 2020; revised November 2020; accepted November 2020 